\title{
Auflehnung und Vermittlung. Petru Dumitrius Essay Die Transmoderne. Zur Situation des Romans (1965)
}

\section{Matthias BAUER}

Prof. Dr., Europa -Universität Flensburg. E-Mail: Matthias.Bauer@uni-flensburg.de

\begin{abstract}
This paper aims to reveal the relevance of a so far neclected essay, written by Petru Dumitriu in German and originally published in 1965 . This essay discussed the situation of the novel and argue against some shortcomings in contemporary conceptions of literature.
\end{abstract}

Key words: nouveau roman, anecdote, structure, diagram(matic)s.

Der Schriftsteller Petru Dumitriu - geboren am 8. Mai 1924 in Baziaş - entstammte einer Familie, in der neben Rumänisch auch Ungarisch und Französisch gesprochen wurde. Er selbst lernte zudem Deutsch. Nach einem Studium der Philosophie in München, das er nach dem Frontwechsel am 23. August 1944 abbrechen musste, avancierte er in der Republica Populară Română zu einer hochdekorierten und einflussreichen Persönlichkeit: Nachdem er 1949, 1952 und 1955 den Rumänischen Staatspreis für Literatur erhalten hatte, wurde er 1956 Direktor des Staatsverlags für Literatur in Bukarest und 1958 Präsident des Verlagsrates beim rumänischen Kulturministerium. Doch ein Jahr nachdem er 1959 mit dem Stern der Volksrepublik Rumänien ausgezeichnet worden war, floh Dumitriu in die Bundesrepublik Deutschland und lebte dann bis zu seinem Tod am 6. April 2002 in Metz. Erst sechs Jahre zuvor, 36 Jahre nach seiner Flucht und sieben Jahre nach dem Sturz Ceauşcescus war er 
erstmalig nach Rumänien gereist. Sein Nachlass befindet sich dank der Vermittlung Elie Wiesels in Cambridge (USA).

Dumitriu hat zu Lebzeiten mindestens zwölf umfangreiche Romane veröffentlicht, darunter das zweibändige Werk Die Bojaren (Bijuterii de familie 1949ff, Cronica de familie 1957; Filmadaption 1957, auf Deutsch nach der französischen Übersetzung 1959/1960 Teil I: Der Familienschmuck, 1960; Teil II: Freuden der Jugend, 1962), mit dem er sich das Misstrauen der Nomenklatura zuzog. ${ }^{1}$ Wie es in diesen Kreisen zuging, wie schnell man dort zum Opfer einer Machtintrige werden und in Ungnade fallen konnte, hat er nach seiner Flucht in dem autobiographischen Schlüsselroman Treffpunkt Jüngstes Gericht (1962) geschildert. Die Erstausgabe erschien ein Jahr zuvor auf Französisch. Auf Deutsch geschrieben wurde zwischen März 1963 und November 1964 das Essay Die Transmoderne. Zur Situation des Romans, der drei Teile umfasst: A. Dialektik der Auflehnung, B. Die Erzählung als Funktion und C. Zum Begriff der Transmoderne. Explizite Bezugspunkte der poetologischen Auseinandersetzung, die Dumitriu in diesem Essay führt, sind Jean-Paul Sartres Konzept der ,littératureengagée ' sowie die Idee des, nouveauroman'. Implizit bezieht er sich aber auch auf das Buch $L$ ' hommerévolté (1951) von Albert Camus. Zur Begründung der eigenen Position, die Camus näher steht als Sartre oder Alain Robbe-Grillet, Michel Simon, Michel Butor und Nathalie Sarraute, rekurriert Dumitriu aufLudwig Wittgensteins bereits 1921 veröffentlichten Tractatuslogico-philosophicus. Indem er die in diesem Traktat entworfenen Sprach- und

Vgl. Laza, Laura Gabriela: „Baumeister war die Angst“. Die politischen Prozesse rumänischer und deutschsprachiger Schriftsteller aus Rumänien nach dem Ungarnaufstand von 1956. Cluj-Napoca: Casa Cărţii de Ştiinţă, 2017, S. 68: Ins „Visier der Kulturpolitiker“ sei Dumitriu dadurch geraten, dass er , in seinem Roman Cronică de familie (Die Bojaren) die Geschichte jener Großgrundbesitzer-Familie bis in 1950er Jahre verfolgt und dadurch problematische Aspekte wie die Enteignung und die politische Verfolgung anschneidet.“ 
Erkenntnistheorie zur Grundlage seiner eigenen, pragmatischen Auffassung vom Erzählen, von dessen Sprachgestalt und Bedeutungsgehalt macht, entwickelt Dumitriu - ohne den Begriff selbst zu verwenden, also implizit - eine regelgerechte ,Diagrammatik' des Romans. ${ }^{2}$. Im Folgenden fasse ich die Kernaussagen von Dumitrius Essay zusammen. Zuvor jedoch einige Bemerkungen zur historischen Kontextualisierung:

\section{Zur Situation des Romans Anfang der 1960er Jahre}

1947 hatte Jean-Paul Sartre in Qu'est-ceque la littérature vom Schriftsteller, den er vom Dichter unterschied, unter dem Eindruck der Verbrechen, die während des Zweiten Weltkriegs gegen die Menschlichkeit verübt worden waren, verlangt, dass er sich jederzeit gegen Ungerechtigkeit und für Gerechtigkeit engagiere. Nur dadurch erhalte sein Schreiben einen gesellschaftlichenWert. Nicht engagierte Literatur sei ein Ausweichen vor der Wirklichkeit, vor den politischen Forderungen der Zeit. Bereits 1942 hatte Albert Camus in Le Mythe de Sisyphe das Absurde der modernen Existenz in der Gegenüberstellung des Menschen, der nach dem Sinn des Daseins frage, und der Welt, die zu dieser Frage schweige, gesehen und, ähnlich wie Friedrich Nietzsche vor ihm, den Ansatz der traditionellen Metaphysik kritisiert, den Sinn in etwas Außer- oder Überirdischem, nicht im Diesseits, sondern im Jenseits, in der ,Tiefe' oder in der Abstraktion zu suchen. Überbrückt und aufgehoben werde die absurde Juxtapositionvon Mensch und Welt im Kunstwerk. Denn in der ästhetischen Einstellung auf die Vielfalt der konkreten Erscheinungen, die das Kunstwerk vermittle, bemerke der Mensch,

2 Vgl. Bauer, Matthias:Romantheorie und Erzählforschung. Eine Einführung. 2., aktualisierte und erweiterte Auflage. Stuttgart / Weimar: Metzler Verlag, 2005, S. 63-70. 
daß die innere Bewegung, die uns vor den Gesichternder Welt hinreißt, nicht von der Tiefe der Welt, sondern von der Mannigfaltigkeit dieser Gesichter herrührt. Die Auslegung ist vergänglich, aber der sinnliche Eindruck bleibt und mit ihm die unaufhörlichen Anrufe eines quantitativ unerschöpflichen Universums. Hier, begreift man, liegt der Ort des Kunstwerks. ${ }^{3}$

In L'hommerévolté wiederum opponierte Camus gegen jede Ideologie und Geschichtsteleologie, die eine Ausgrenzung, Unterdrückung oder gar Vernichtung von Menschen um eines angeblich höheren Zweckes willen rechtfertigt. Er hatte dabei sowohl den italienischen Faschismus und den Nationalsozialismus in Deutschland als auch den Totalitarismus unter Stalin im Blick. Man müsse sich gegen jede Form des rigiden Denkens und Handelns, das nur Freund und Feind kenne, auflehnen. Diese Revolte entstehe nicht unbedingt im Unterdrückten selbst, sondern in jedem Menschen, der Zeuge von Unterdrückung werde. Da Camus bei seiner umfassenden Kritik keinen Unterschied zwischen der Unterdrückung im Namen rechter und der Unterdrückung im Namen linker Weltanschauungen machte, wurde er von Sartre und anderen marxistisch gesinnten Intellektuellen harsch attackiert.

Eine andere Auffassung von ,Revolte' liegt der Moderne, insbesondere den Modernismen der Avantgarde zugrunde, die jeweilsmit einer Auflehnung gegen Tradition und Konvention begonnenhaben. Auch hier geht es um eine ästhetische Einstellung, die ineinigen Ausprägungen zu einer Absage an eine sinnhafte Vermittlung von Mensch und Welt, Individuum und Kollektiv geführt hat und insofern sowohl den Vorstellungen von Sartre alsauch von Camus widerstreitet. Ein seinerzeit viel beachteter Exponent dieser Revolte gegenjede Form der

3 Camus, Albert Der Mythos des Sisyphos. Ein Versuch über das Absurde. Mit einem kommentierenden Essay von Liselotte Richter. Übertragen von Hans Georg Brenner und Wolfdietrich Rasch. Reinbek bei Hamburg: Rowohlt, 1984 [1942], S. 80. 
Fremdbestimmung von Kunst war der ,nouveauroman' in seinem Bestreben nach künstlerischer Autonomie. Diese Autonomie sollte sich im Rück(be)zug der Literatur auf ihr Material, auf die Sprache, sowie im Verzicht auf konventionelle Figurenund Handlungskonzepte kundtun, denen man im Zeitalter des Argwohns - so der Titel eines einflussreichen Essays von Nathalie Sarraute - nicht mehr trauen könne.

\section{A. Dialektik der Auflehnung}

Vergegenwärtigt man sich die Situation des Romans zu Beginn der Sechzigerjahre anhand eines Dreiecks, das zwischen den Positionen von Sartre, Camus und den Vertretern des ,nouveauroman' aufgespannt wurde, die damals weit über Frankreich hinaus Beachtung fanden, lässt sich Dumitrius Essay zugleich als ein Versuch der Auflehnung und der Vermittlung lesen. Einerseits begehrt er gegen die vergleichsweise radikale Idee des ,nouveauroman' und die nicht minder radikale Vorstellung von einer der Moderne eingeschriebenen, permanenten ästhetischen Erneuerung auf - andererseits vermittelt er die Poetik des realistischen Erzählens, die sich mindestens bis auf Stendhal zurückführen lässt, mit dem Anspruch, aufschlussreiche, der Gegenwart verpflichtete und gesellschaftsrelevante Literatur zu verfassen. Folgerichtig heißt es im ersten Teil des Essays unter der Überschrift ,Dialektik der Auflehnung ${ }^{64}$ : ,Revolte ist nichts, wenn sie nicht erneuert wird.“ (7) Und weiter:

Die Wiederholung der Revolte in der letzten Zeit hat eine bisherunbekannte Lage geschaffen, in der sich der Künstler gegen die Tradition, das heißt gegen die Gesellschaft, aufzulehnen hatte, um seinem Individuationstrieb zu genügen, anders gesagt, umdas, was man seinen existentiellen Entwurf genannt hat, zu verwirklichen [...]. (7)

${ }^{4}$ Petru Dumitriu. Petru:Die Transmoderne. Zur Situation des Romans. Frankfurt am Main: S. Fischer Verlag, 1965 erfolgen gemäß dieser Ausgabe direkt im Text. 
Interessant an dieser Bemerkung ist zum einen, dass die Gesellschaft für Dumitriu durch ihre Tradition(en) bestimmt wird. Sie ist, so ließe sich der Gedanke ausführen, wie die Sprache, ohne die sie nicht auskommen kann, eine auf Konventionen beruhende Einrichtung, die es somit genealogisch, anhand ihrer historischen Entwicklung - und das meint in erster Linie: anhand der Überlieferungen, auf die sie sich beruft - zu verstehen gilt. Wichtig ist zum anderen, dass der wiederholte Traditionsbruch, der die Moderne auszeichnet, aus seiner Sicht in der zeitgenössischen Kunst selbst zu einer Konvention geworden ist. Progressiv könne ein Künstler nur noch sein, wenn er sich von den sozialen und sprachlichen Konventionen, den Formen der Überlieferung und dem Sinnvorrat der Tradition abhebe. Literatur, die diesen Namen verdiene, könne daher angeblich ,nur engagiert oder autonom sein, nur erneuernd oder traditionell. Tertium non datur. Das Folgende ist eine Untersuchung, ob es doch eine andere Lösung gibt.“ (10) Um diese ,andere Lösung' zu erreichen, habe ,sich literarische Opposition heute gegen die manichäische Denkweiseaufzulehnen“"(12), die für Dumitriu auf eine einfache Formel hinausläuft:

$[\ldots]$ die Welt $=$ Freund + Feind; derFeind $=$ nichts. Es ist der pathologische Wunsch, die Welt auf dasIch und das dem Ich Gleichartige zu reduzieren sowie eine Werttafel zu entwerfen, auf der das eigene Ich und seinesgleichenden höchsten Rang einnehmen würden. Der selbstzufriedene Manichäismus begegnet uns auf Schritt und Tritt in den literarischen Zeitschriften. „Ich glaube, der ,neue Roman“ ist die heuteeinzige Form des Berichtens", sagt Michel Butor in einem Interview (,Die Welt, 6. 3. 1964); „Keine aufrichtige realistische Tendenz kann sich damit begnügen, eine banale Wirklichkeit zu erforschen und sie in traditioneller Form wiedererstehen zu lassen", sagt Nathalie Sarraute (,Lettresnouvelles', Febr-März 1964, S. 97) [etc.]. (13)

Damit sind die Fronten klar. Dumitriu wendet sich in seinem Essay gegen jene Konzeption des literarischen Werks, die neben der eigenen, angeblich einzig echten und allein richtigen 
keine andere Form des Berichtens zulässt. Dieser Behauptung gegenüber vertritter die,,Antithese [...], daßmehrere Richtungen legitim, mehrere Richtungen echt und aufrichtig sind, daß es keine vereinzelte richtige Konzeption gibt.“ (14) Eine solche, dem Gedanken des Pluralismus verhaftete Auffassung vom Schreiben und Lesen muss nicht nur Einspruch gegen die Idee des ,nouveauroman', sondern auch gegen das Konzept der ,littératureengagée' erheben. Dieses Konzept kulminiert für Dumitriu in der Aufhebung von Literatur.,,, Was bedeutet Literatur in einer Welt, in der man hungert?', fragt Sartre. Die Antwort ist fraglos ,Nichts!““(22) Gegen diese radikale Infragestellung aller Literatur wendet Dumitriu, der sich damit auf die Seite von Camus schlägt, ein:

Vor dem Angesicht des Bösen in der Welt schrumpfen alle unsereWerte wie brennendes Papier zusammen. Aber wer mit dieserWahrheit vor Augen lebt, kann nur entweder den Selbstmordoder den Weg des eremitischen oder tätigen Mystikers gehen;was seine Sprache betrifft, kann sie nur Predigt sein oder Schweigen. Keines von beiden ist Literatur. (22 f.)

Markiert wird mit diesem Argument nicht nur ein dezidiert ästhetischer Standpunkt, sondern wie in L'hommerevolté ein kritischer Blick auf alle Weltanschauungen, die dem Freund-FeindSchema verhaftet sind. Scheint nämlich, wie Dumitriu schreibt, „,der Grundirrtum der manichäischen Denkweisedarin zu bestehen, daß sie aus der Mannigfaltigkeit des Kunstwerkes entweder den einen oder den anderen Aspekt herausreißenund ihn zu einer falschen Totalität erheben will“" (33), so ist von dieser Kritik auch jede politische Handlung betroffen, die aus dem Meinungsspektrum der Gesellschaft diese oder jene Position isoliert, zur Totalität erhebt und zunächst auf die Exklusion aller abweichenden Vorstellungen, in letzter Konsequenz aber, wie unter Hitler und Stalin geschehen, auf die Vernichtung aller Menschen hinausläuft, die solche Vorstellungen hegen oder auch nur hegen könnten. Eingedenk des dictum horribile, das 
Hegel zugeschrieben wird - Wenn die Idee nicht zur Wirklichkeit passt, umso schlimmer für die Wirklichkeit ${ }^{6}$ - und im Bewusstsein der historischen Katastrophen, die das ideologische Handeln im 20. Jahrhundert heraufbeschworen hat, kann man daher mit Dumitriu feststellen, „daß die Vielgesichtigkeit der Realitätnicht von der manichäischen Denkweise erfaßt zu werden vermag. Es ist aber ebenso augenscheinlich, daß diese Realität vorhanden, daß sie schillernd, mannigfach, widersprüchlich ist.“ (86)

$\mathrm{Zu}$ dieser Widersprüchlichkeit der Wirklichkeit gehört, dass sie sowohl durch Erneuerung als auch durch Überlieferung, durch Innovation wie durch Tradition und eben nicht ausschließlich durch permanente Revolutionen geprägt ist. Dass Dumitriu gerade unter diesem Gesichtspunkt das Politische und das Ästhetische zusammen denkt, erhellt aus seiner Bemerkung: „Eine fünfzig oder sechzig Jahre alte Kunstbewegung ist so wenigeine Avantgarde, wie ein fünfzig Jahre alter Machtstaat noch revolutionär sein kann.“ (90) Entsprechend wird die Transmodernevon Dumitriuwederals Steigerung, als Radikalisierung, noch als Negation der Moderne ins Auge gefasst. Er lehne die Moderne „nicht zugunsten der Tradition, sondern zugunsten von etwas ab, das sie beide überbrückt undihren unversöhnlichen Gegensatz im Grenzfall paradox in sichaufnimmt.“ (94)

\section{B. Die Erzählung als Funktion}

Wie eine solche Vermittlung, eine solche Moderation gelingen kann, exemplifiziert Dumitriu im zweiten Teil seines Essays exemplarisch mit Blick auf die Erzählkunst. Ausgangspunkt ist die Beobachtung, dass der Roman in seiner Eigenschaft als Bericht (fiktiver Ereignisse) nicht im Gegensatz, sondern im Einvernehmen mit der allgemeinen Sprechtätigkeit des Menschen steht: 
In der Tat können wir uns kaum einige Zeit mit einem anderen Menschen unterhalten, ohne dabei mindestens Bruchstücke von Berichten zu liefern. Weiter noch: es ist kaum möglich, außer alsdurch äußerste Willensanstrengung und Konzentration der Aufmerksamkeit, einen Bericht zu liefern, der von unseren eigenen,im Gedächtnis gespeicherten Informationen nicht in irgendeinem Punkt abweichend wäre. [...] Unser Bericht wählt aus, vereinfacht, verdichtet, gliedert, verarbeitet also Gedächtnismaterial, das schon verarbeitetes Wahrnehmungsmaterial ist. (129f.)

Nicht erst im Bereich der Dichtung, schon in der Alltagskonversation kommt es, so gesehen, zu Konfabulationen und Interpolationen - also zu dem, was Hayden White später, emplotment' genannt hat ${ }^{5}$ und $\mathrm{zu}$ jenem Überschreiten der empirischen Realität durch die Imagination, an dem Wolfgang Iser seinerseits die Funktionen des Fiktiven festgemacht hat ${ }^{6}$. Unterlaufen wird damit auch die Legende von einer poetischen Sprache, die grundsätzlich anders als die normale Ausdrucksweise der zwischenmenschlichen Verständigung sei. Diese schon im Russischen Formalismus ${ }^{7}$ und auch später im 20. Jahrhundert immer wieder virulente These koppelt die Literatur von der gesellschaftlichen Konstruktion der Wirklichkeit ab und erkauft ihre Autonomie mit ihrer Irrelevanz. Sie ist für Dumitriu inakzeptabel. Im Gegenzug verweist er auf die Verwandtschaft zwischen Tagebuch und Geschichtsschreibung einerseits und Roman und Drama andererseits (vgl. 137) und schließt aus dieser Verwandtschaft:

5 Vgl. White, Hayden: Auch Klio dichtet oder Die Fiktion des Faktischen. Studien zur Tropologie des historischen Diskurses. Übersetzt von Brigitte Brinkmann-Siepmann, Vorwort von Reinhart Koselleck. Stuttgart: Klett, 1986.

6 Vgl. Iser, Wolfgang: ,Akte des Fingieren oder Was ist das Fiktive im fiktionalen Text?", in: Funktionen des Fiktiven, hrsg. v. Dieter Henrich und Wolfgang Iser. München: Fink-Verlag, 1983, S. 121-151.

7 Vgl. Bauer, Romantheorie und Erzählforschung, S. 109. 
Wir haben also nicht nur Abgrenzungen vorzunehmen, sondern Übergänge und Kommunikationsgebiete zu suchen oder zu schaffen, wenn unsere Untersuchung nicht nur auf der Erkenntnis der bisherigen Erzählungen beruhen, sondern auch die Schaffung von Grundbedingungen zukünftiger Fiktion anstreben soll. (136)

Im gleichen Zusammenhang verweist er auf jenen Übergang vom Tatsachenbericht zur Fiktion im Schlüsselroman (vgl. 138), den man in geradezu paradigmatischer Form an Treffpunkt Jüngstes Gericht studieren könnte. Unvereinbar ist diese Idee einer diskursiven Vermittlung von Fiktion und Realität, von ästhetischer und politischer Praxis freilich mit der Vorstellung, ein Roman ,sei nichts weiter als ein Sprachkörper, eine Aneinanderreihung von Sätzen. Aus ihren Besonderheiten, aus ihrer Beschaffenheit könne man die Eigenart des Werkes und des Dichters herauslesen.“ (147) Genau diese Vorstellung verbindet für Dumitriudie Vertreter des ,nouveauroman' mit anderen, weniger avantgardistisch verfahrenden Schriftstellern der Gegenwart. Sie alle neigten dazu, ,das meiste, was nicht sprachlich verifizierbar ist, bei der Untersuchung eines Romans zu übersehen.“ (147) Insbesondere werde jene Schicht im Sprachkunstwerk übersehen oder unterschätzt, die Alain RobbeGrillet verächtlich ,Anekdote' nenne, obwohl sich gerade an ihr das stets besondere Ingenium des Autors und zugleich, so darf man Dumitriu verstehen, die allgemeine Relevanz oder Übertragbarkeit der erzählten Geschichte auf andere Zusammenhänge, nicht zuletzt auf die vorherrschende Lebenswirklichkeit der Leser, zeige. Daher behauptet Dumitriu zum einen,

daß nur Stendhal die Anekdote von ,Rot und Schwarz', nur Balzac dieAnekdote von, Glanz und Elend der Kurtisanen“, nur Dostojewskij die Anekdote von ,Die Brüder Karamasow', nur Kafka die Anekdote von ,Das Schloß` erfinden konnten und daß in solchen Anekdoten ein gut Teil der Bedeutung solcher Werke liegt. (166)

Zum anderen stellt er mit der gleichen Entschiedenheit fest: „Die Anekdote ist wichtig, denn das Wirklichkeitsbild, welche 
sein solches Gefüge von Handlungen und Situationen entwirft, ist sinnvoll.“ (166) Mit diesem Satz kommt er zum Kern seiner eigenen Poetik, zu einer Auffassung vom Erzählen, die man wie weiter unten näher ausgeführt wird - als , diagrammatisch ${ }^{6}$ bezeichnen kann. Aus Gründen der Verständlichkeit soll es vorerst aber nicht um diese semiologische Implikation der ,Anekdote', sondern um den Nachvollzug der Argumentation gehen, die Dumitriu in seinem Essay ausdrücklich entfaltet. Gilt nämlich, das spezifisch „Kafkasche, das Dostojewskijhafte eignet den erzählten Begebenheiten, ihrer Zusammensetzung, den beschriebenen Menschen, ihrem Betragen und ihrem Schicksal“" (148), so kann man generell sagen:

Der Sprachkörper ist einer der Bestandteile des Romans; er ist seine äußerste Schicht, seine erste Ebene, seine Haut, ohne die wir niemals mit dem Roman in Berührung kämen. Es gibt aber mindestens eine zweite Schicht, und das ist das Gerippe der Handlung, welche einen gewissen Sinn hat [...]. Diese zweite Stufe ist eine Sprache der Beziehungen und Charaktere, eine Syntax des Schicksals. (151)

Die Pointe der ,Anekdote' liegt somit darin, dass sie ein Beziehungsmuster veranschaulicht. Ebenso besteht der Clou des Romans darin, „daß sich seine Gestalten in bestimmter Art und Weise zueinander verhalten. “(168) Insofern die erzählte Handlung „,eine Konfiguration von Gebärden und Reden in der Zeit"“ (195) darstellt, erlaubt sie Analogieschlüsse. Aus der TextLektüre, aus der sukzessiven, konjekturalen Erfassung der Ereigniszusammenhänge, wird dergestalt ein Inferenzprozess - ganz so, wie Paul Ricœur den Übergang von der narrativen Konfiguration der Fabel zu ihrer Refiguration durch die Leser im Lichte ihrer eigenen Erfahrung aufgefasst hat. ${ }^{8}$ Was Dumitriu auf seine Weise und Ricœur in anderer Form konstatieren, lässt sich im Übrigen bis auf Aristoteles und seine Auffassung vom

8 Vgl. Ricœur, Paul:Zeit und Erzählung. Band I: Zeit und historische Erzählung. Aus dem Französischen von Rainer Rochlitz. München: Fink, 2. Aufl. 2007., S. 120-122. 
,mythus' als einer folgerichtig komponierten Abfolge von Begebenheiten zurückführen, deren Verlauf den Zuschauern im Theater (wie den Lesern eines Romans) bestimmte Schlussfolgerungen auf die so genannte Lebenswelt erlauben. Anders gesagt: Die Anekdote respektive der ,mythus' ist als eine Art Schaubild, als Beziehungsgefügezu begreifen und fungiert in dieser Hinsicht als Modell für die Auslegung analoger Ereigniszusammenhänge oder Handlungsoptionen in der Realität, kurzum als Diagramm. Tatsächlich behauptet Dumitriu denn auch explizit: „Das Bild ist ein Modell der Wirklichkeit“, und fügt sogleich hinzu: „Es ist aber nicht von unserer Praxis unabhängig.“ (168)

Das eine - der Modellcharakter der Literatur - ist vom anderen - von ihrer pragmatischen Bedeutung - nicht zu trennen. Und eben darin liegt die Vermittlung von Poetik und Politik, um deren Willen sich Dumitriu gegen die Abkopplung der ästhetischen Tätigkeit von allen anderen Handlungen im Zeichen einer fragwürdigen Autonomie des Kunstwerkes auflehnt. Gegen die Auffassung von der gänzlich zweckfreien Kunst setzt er die Vorstellung, dass Dramen oder Romane die Aufgabe haben, sinnliche Anschauung, Einfühlung und Erkenntnis zusammenzuführen. Da „die Empathie nur im Konkreten möglich ist, die abstrakte, allgemeine, begriffliche Erkenntnis als Akt also die Empathie ausschließt" (169), muss das Kunstwerk - in dieser Hinsicht liegt Dumitriu ganz auf der Linie von Camus - die Vielfalt der Erscheinungen vergegenwärtigen. Mehr noch: für Dumitriu wird durch das „stellvertretende Erleben“ (170), das sich in der Romanlektüre vollzieht, wenn der Leser mit den Helden der Geschichte fühlt, eine „Gemeinschaft“ (170). Zugleich übersteigt das stellvertretende Erleben die auf Erfahrung beruhende Erwartung. Es läuft, so verstanden, auf eine Transfiguration der empirischen Wirklichkeit hinaus.

Denn Leben, das beginnt man heute zu erkennen, ist zugleich einMehrerlebenwollen, ein Expansionsdrang des Individuums wie der 
Gruppe. Dieser Drang verklärt sich im stellvertretenden Erleben: vicariousness, ja Kunst überhaupt, jedenfalls Epik und Drama, wären demnach die Sublimierung des potentiell weltzerstörerischen Expansionsdranges des Subjekts. (171)

Man könnte in dieser Moderation des individuellen Expansionsdrangs durch das Kunstwerk eine Replik auf Nietzsches, Willen zur Macht' sehen. Dieser Wille bekundet sich vor allem darin, dass der Mensch bestrebt ist, seine Kraft, die zugleich eine gestalterische und eine zerstörerische Komponente hat, an der Welt auslassen - was immer dann, wenn diese Kraft nicht ästhetisch gebändigt wird, zu gewaltsamen Umwälzungen führt. Das stellvertretende Erleben und Ausleben, das Leseabenteuer gestatten, erweist sich demgegenüber, insofern man es als Sublimation des Expansionsdranges - freudianisch gesprochen: des Aggressionstriebes - betrachten kann, als eine Kulturleistung derLiteratur, die der Gemeinschaftzugutekommt. Voraussetzung ist allerdings, dass die Agenten der Romanhandlung - sozusagen die Avatare der Leser - tatsächlich handeln und eben das produzieren, was man ,Drama', ,Anekdote‘ oder ,mythus' nennt. Auf genau diesen Zusammenhäng beziehen sich denn auch Dumitrius Überlegungen zur Erscheinungsgestalt des Helden:

Der vollkommen irrationale Held ist ebenso unerträglich und unglaubwürdig wie der vollkommen berechenbare; die vollkommengestaltlose Handlung - schon im Ausdruck widersprüchlich, denn Handlung ist eben eine Konfiguration von Gebärden und Reden inder Zeit - ist ebenso unglaubwürdig und unbefriedigend wie dievollkommen voraussehbare Handlung. In diesem Sinne können wirbei aller Anerkennung ihres grundsätzlichen Rechtes auf literarisches Dasein, bei allem Verständnis der individuellen und kollektiven Ursachen ihres Entstehens, sowohl die modernen westeuropäischen Romane mit gestaltlosen dramatispersonae als auch densogenannten sozialistischen Realismus mitseinen unfehlbarenParteisekretären, der immer weisen und gerechten Partei und dem unfehlbaren Triumph der guten Sache am Ende, auf Grund unserer Erkenntnis des Menschen nur ablehnen. (195) 
Letztlich entscheidend ist daher nicht das Abenteuer, welches dem Romanhelden widerfährt, sondern die Gelegenheit, die es dem Leser verschafft, sich in der Kunst der Menschenkenntnis zu üben, denn:

Instinktiv sind wir auf einer Mikro- oder Makro-Ebene jede Sekunde und auch ein ganzes Leben hindurch bestrebt, unsere Mitmenschen zu erkennen. Selbst wenn wir es gar nicht wollen, müssenwir Peilungen durchführen, Charakter- und Beziehungsmodellevon unseren Mitmenschen entwerfen, um einfach mit ihnen zusammenleben zu können. (207)

Exakt hier liegt für Dumitriu der Treffpunkt von ästhetischer und sozialer, von poetischer und politischer Tätigkeit. Als Spezialfall dessen, was der Roman als Medium der Erkenntnis leisten kann, erscheint in diesem Zusammenhang der Kriminalroman. Die Schilderung der Täterermittlung, die seine Handlung zur Hauptsache ausmacht, ist nämlich ,nichts anderes als die Beschreibung eines derartigen Orientierungsverfahrens. Man orientiertsich über eine Menschengruppe: wer darunter ist der Mörder?" (207) Die Spannung, die ein Krimi beim Leser erzeugt, beruht demnach auf der Ungewissheit darüber, welche seiner Gestalten ein Verbrecher ist. Im Unterschied zu dieser, an den Ausnahmefall des Mordes gebundenen Ungewissheit, „ist der Roman, ist die Großerzählung unter all ihren Namen auf einer viel allgemeineren und differenzierteren Ungewißheit aufgebaut, nämlich auf der Ungewißheit über die Struktur der Welt und der sie bevölkerndenMenschen." (210)

Diese Ungewissheit wird im Akt des Lesens gleichsam hypothetisch aufgehoben unter der Voraussetzung, dass die Narration nicht nur Ungewissheit provoziert, sondern auch Deutungsmuster evoziert. Werden der rezeptiven Einbildungskraft nämlich nicht nur Leerstellen, sondern auch Schemata zu ihrer Überbrückung offeriert, erweist sich der Akt des Lesens als ein kreativer Prozess. Dieser, wie man heute sagen würde, performativen Dimension der Interaktion von Text und Leser ist 
Dumitriu auf der Spur, wenn er sich den Schlachtbeschreibungen bei Stendhal oder Tolstoi zuwendet und nach dem „Strukturschema in dem ganzen Geschehen" (217) fragt. Inwiefern ist die Schlachtbeschreibung ein Schaubild menschlichen Handelns, ein Diagramm ,der Bezugsrelationen, an denen der Mensch beteiligt seinkann"(217)? Keineswegs wird durch die Entdeckung bedeutsamer, auslegungsrelevanter und signifikanter Relationen und Strukturen, ausgeblendet, dass die Realität absurde Züge hat und selten auf Anhieb Sinn macht:

Wir wollen keineswegs die meist chaotische Oberfläche der Wirklichkeit ableugnen. Wir wollen nur jenes Dogma entschieden ablehnen, das diese Oberflächenerscheinung der Wirklichkeit, unverständlich, rätselhaft, wie sie beim ersten reflexionslosen Blick auch ist, als die Wirklichkeit schlechthin erklärt. (218)

Unter dieser Einschränkung gelangt Dumitriu am Ende des zweiten Teils seines Essays zu einer Modifikation der Entfremdung zwischen dem Menschen, der nach dem Sinn seiner Existenz fragt, und einer Welt, die ihm die Antwort schuldig bleibt. Schon Camus kam es darauf an, diese vermeintlich statische Gegenüberstellung in eine dynamische Beziehung zu überführen: durch die künstlerische Tätigkeit, die sich auf die Vielfalt der sinnlichen Erscheinungen kapriziert, wird die schweigende Welt in ein Universum der unaufhörlichen Anrufe verwandelt, wird dort, wo es scheinbar gar keinen Widerhall gibt, ein Resonanzraum geschaffen. Zu klären bleibt, ob und wie sich dieser Resonanzraum auch jenseits der ästhetischen Tätigkeit und der spezifischen Dynamik (Wirksamkeit), die sie entfaltet, mit der Lebenswelt in Beziehung setzen lässt. Auf diese Frage gibt es wenigstens zwei Antworten: die eine läuft auf eine durchgehende Ästhetisierung der Lebenswelt, auf das romantische Projekt einer Universalpoesie hinaus, an das die künstlerische Avantgarde der Moderne immer wieder angeknüpft hat - etwa dadurch, dass Gegenstände des alltäglichen 
Gebrauchs als Kunstwerke inszeniert wurden (readymades). Dumitriu stellt und beantwortet die Frage etwas anders:

Warum sind wir Fremde in dieser Welt, warum ist diese Weltuns fremd? Aus keinem anderen Grund als dem, daß wir selbst die Brücken zwischen ihr und uns abgebrochen haben, jene Brücken, die die Praxis schlägt, die unser handelndes, wirkendes Ich in die Wirklichkeit projiziert und womit sie diese abtastet, durchdringt, sich aneignet. (219)

Der Brückenschlag, der ihm vorschwebt, setzt weniger auf die ästhetische Transfiguration der Welt durch romantische Vorstellungen und damit auf jene Magie der Einbildungskraft, die Gefahr läuft, sich im Zeichenzauber der Sprache zu erschöpfen. Er setzt vielmehr auf die Modellfunktion, die der Erzählung als Handlungsbericht zukommt. Wenn die Struktur der Welt und die Struktur der Fabel so beschaffen sind, dass der Text Rückschlüsse auf die Wirklichkeit erlaubt und der Romanleser das handelnde Ich, als das er sich im empathischen Nachvollzug der (fiktiven) Anekdote imaginiert, in die empirische Welt projizieren kann, erhält die Tätigkeit, die er realiter entfaltet, eine Wirksamkeit, die dem Gefühl der Sinn- und Machtlosigkeit und damit der Entfremdung von Mensch und Welt entgegenarbeitet. Zu bedenken ist dabei stets, dass jene Form der kreativen Aneignung von Handlungsmacht, die ein Roman ermöglicht, an seine Welthaltigkeit gebunden ist. Trennt der Schriftsteller jedochdie Arbeit an der Sprache von der Mitarbeit an der gesellschaftlichen Konstruktion der Wirklichkeit, geht mit dem Zusammenhang zwischen ästhetischer und sozialer Praxis auch der Zusammenhang zwischen poetischer Kraft und politischer Macht verloren, wird das Absurde nur reproduziert und nicht überwunden. 


\section{Zum Begriff der Transmoderne [und zur Diagrammatik des Romans]}

Angesichts dessen, was Dumitriu im ersten und im zweiten Teil seines Essays ausführt, nimmt es nicht wunder, wie er den genealogischen Zusammenhang von Moderne und Romantik rekonstruiert, um im dritten Teil ,Zum Begriff der Transmoderne“ vorzustoßen: ,Die Moderne oder Avantgarde und ihre rechtmäßige Mutter, dieRomantik, haben sich vor allem als Auflehnung gegen die literarische und künstlerische Konvention definiert.“ (96) Im Ergebnis habe diese Auflehnung zu der Verlassenheit und Vereinsamung des Menschen geführt, die dem Eindruck, in einer absurden Welt zu leben, zugrundeliege. (vgl. 253) Dieser Eindruck ist nichts anderes als der Eindruck der Entfremdung. Doch, so lautet Dumitrius Credo:

Unser Grundempfinden der Welt gegenüber ist anders. Auchunsere Grunderfahrung der Welt ist verschieden. [...] Wir erkennen uns wieder in der Handhabung der Werkzeuge, der Waffen, der wissenschaftlichen Methode, in der Anwendungder technischen Verfahren. Deswegen ist die Welt für uns voller Zeichen, die uns mögliche Bezugnahmen offenbaren. (254)

Um eine Welt voller Zeichen, die Bezugnahmen ermöglichen, zu erhalten, muss man, semiologisch formuliert, eine Operation der Aneigung vollziehen, die zu allererst einmal darin besteht, die Welt als ein Beziehungsgefüge, also diagrammatisch, aufzufassen. Das damit verbundene Menschenbild ist das eines Operateurs,

der Wirklichkeitsmodelle, Wirkungsmodelle, Aktionspläne, Funktionsschemata, Ereignistabellen konstruiert ${ }^{9}$ und den Wittgenstein,

9 Nicht umsonst erinnert diese Formulierung an eine Stelle in Robert Musils „Skizze der Erkenntnis des Dichters“ (1918), an der es heißt: „Die Aufgabe ist: immer neue Lösungen, Zusammenhänge, Konstellationen, Variable zu entdecken, Prototypen von Geschehensabläufen hinzustellen, lockende Vorbilder, wie man Mensch sein kann, den 
ebenfalls auf der Grenze zwischen später Moderne und zu seiner Zeitnoch nicht entstandener Transmoderne, beinahe erschöpfenddefiniert hat, als er zum 15. April 1926 in sein Tagebuch notierte: ,Nur was wir selbst konstruieren, können wir auch verstehen. ' (257)

Wittgenstein steht mit dieser Definition in der Tradition von Kant, für den der Verstand nur begreifen kann, was sich gemäß der Verfahren, über die er selbst verfügt, herstellen lässt. Dumitriu ratifiziert Wittgensteins Erkenntnistheorie, wenn er den Menschen, das dezentrierte Subjekt der Moderne, wieder in der Mitte der Welt stellt - wenn auch, ,nur insofern, als die Welt keine objektive Mitte mehr, wohl aber eine operative Mitte hat." (258) Selbst wenn man diese Lokalisierung des Menschen inmitten der Welt nur als heuristische Fiktion betrachtet, hat sie unter dem Gesichtspunkt der, agency“ eine pragmatische Bedeutung. Zu ihren Konsequenzen gehört nicht zuletzt eine dynamische Auffassung der Welt, die man auch, historisch“ nennen könnte: Was ist, wurde gemacht und kann durch Handlungen geändert werden. Es wird, mit anderen Worten, ,stets darum gehen, die Wirklichkeit in Fluß zu halten und unser Verständnis immerfort dialektisch (also jeweils ablehnend und aneignend) diesem Fließen nachzubilden." (17) Die von den Vertretern des ,nouveauroman' verachtete und von Dumitriu rehabilitierte Anekdote wäre, so verstanden, zugleich der Gegenstand, den der Leser dialektisch verstehen soll, und, mit Charles Sanders Peirce zu reden, das unmittelbare Objekt der Zeichendeutung, an dem sich die Prozessstrukturen des dynamischen Objekts schlechthin, der Welt, offenbart. „Diese Strukturen sind in Bewegung begriffen, dafür aber nicht unkenntlich und unbeschreibbar." (191)

Die Position, die Dumitriu mit dieser Aussage im diskursiven Feld der Poetologie und Epistemologie des 20. Jahrhunderts

inneren Menschen erfinden." (Musil, Robert: Gesammelte Werke in neun Bänden, herausgegeben von Adolf Frisé. Band 8. Essays und Reden. Reinbek bei Hamburg: Rowohlt, 1978, S.1025-1030, hier S. 1029.) 
bezieht, widerstreitet der Annahme, dass die Ordnung der Dinge nicht mit der Ordnung der Worte in Überstimmung zu bringen sei - eine Annahme, die sich für Michel Foucault und andere Denker, scheinbar zwingend, aus der Verabschiedung des Ähnlichkeitsdenkens aus dem wissenschaftlichen Diskurs ergibt, die im 17. Jahrhundert einsetzte und in der radikalen Differenzlogik der Modernen kulminiert. ${ }^{10}$ Dumitriu hingegen hat keine Scheu zu behaupten: „Der Grund der Sprache sind die Dinge und ihre Ordnung. Im Augenblick, in dem man der Sprache auf den Grund zu kommen vermeint, ist man mit den Dingen und ihrer Struktur in Berührung geraten." (105) Woher er diese Überzeugung nimmt, wird deutlich, wenn man seinem Verständnis von ,Text' nachgeht, denn ein Text ist für ihn

[... nicht nur die Summe seiner Worte, sondern auch die Summe seiner direkten und indirekten Bedeutungen. Durch diese Bedeutungen, die ihn ebensosehr ausmachen wie die materiellen Gegebenheiten der Worte, weist dieses Gewebe von Worten auf ein Gewebe von Fakten hin. Die logische Ordnung, sagt Wittgenstein, ist die Ordnung der Welt. Die Art und Weise, in der sich die Worte innerhalb eines Textes zueinander verhalten, entspricht der Art und Weise, in der sich die Dinge, auf die die Worte weisen, zueinander verhalten. (106 f.)

Fast gleichlautend heißt es bei Umberto Eco in Opera aperta (1962; dt. Übersetzung 1977): ,[...] die Literatur organisiert Wörter, die Aspekte der Welt bezeichnen, doch das literarische Werk deutet auf die Welt hin durch die Art und Weise, wie diese Wörter angeordnet werden. " ${ }^{11}$ Auch bei Eco firmiert Wittgenstein als spiritusrector dieser Auffassung, die zugleich eine poetologische und eine epistemologische ist. Stärker als der italienische Semiologe und spätere Romanautor, der das offene Kunstwerk

${ }^{10}$ Vgl. Foucault, Michel: Die Ordnung der Dinge. Eine Archäologie der Humanwissenschaften. Aus dem Französischen von Ulrich Köppen. Frankfurt am Main: Suhrkamp, 1974, S. 46.

${ }^{11}$ Eco, Umberto: Das offene Kunstwerk. Übersetzt von Günter Memmert. Frankfurt am Main: Suhrkamp, 1977 [1962], S. 271. 
rezeptionsästhetisch konzipiert hat, geht Dumitriu in seinem Essay allerdings von der Produktionsästhetik, von der Praxis des Erzählens aus. Gerade die Ordnung der Wörter, die das narrative Gewebe des Romans ausmacht, folgt aus seiner Sicht aus der Notwendigkeit, eine Auswahl treffen zu müssen:

Welcher Erzähler, und sei er auch noch so objektiv, trifft unter seinen Materialien keine Auswahl? Welcher entwirft kein Muster eines bedeutungsvollen Schemas in der Fülle der zu berichtenden Ereignisse. (139 f.)

Was White als ,emplotment" und Ricœur als ,Konfiguration“ bezeichnen, ist für Dumitriu zunächst einmal eine Folge des Selektionszwangs, unter dem jede Erzählung steht. Ordnungsmuster und Deutungsschemata sind ein Produkt dieses Zwanges, in denen zugleich etwas Objektives und etwas Intersubjektives steckt. Das Objektive liegt in der Unvermeidbarkeit der Auswahl, im Rückverweis auf die Komplexität die Welt und die Kontingenz jeder narrativen Ordnung und Schemabildung; die Intersubjektivität aber ist durch den sprachlichen Zuschnitt dieser Ordnung und Schemabildung gegeben. Einerseits kann man daher am Erzähltext ablesen, wie er die Wirklichkeit reduziert und modelliert; andererseits hängt gerade an diesem Aspekt seiner Lesbarkeit ein wesentliches, weil reflexives Erkenntnismoment. Es geht dabei um den perspektivischen Charakter aller Weltbeschreibung und Ereignisschilderung, Figurendarstellung und Sinnaneignung.

Genau auf diesen perspektivischen Charakter hebt Ludwig Wittgenstein im Tractatuslogico-philosophicus ab, wenn er erklärt: „,Wir machen uns Bilder von den Tatsachen.“ (Satz 2.1) ${ }^{12}$ $\mathrm{Zu}$ denken ist dabei primär nicht an (fotografische) Abbilder in der Semiotik, images' genannt - sondern an Schaubilder

12 Wittgenstein, Ludwig: Tractatus Logico-Philosophicus. Logisch-Philosophische Abhandlung. Frankfurt am Main: Suhrkamp, 1962, Zitate werden im Folgenden jeweils nach der Satznummerierung belegt. 
(,diagrams'), die Strukturzusammenhänge, Beziehungsmuster, Verhältnisbestimmungen vor Augen führen. Dumitriu übersetzt den soeben zitierten Satz zusammen mit einer Reihe anderer Äußerungen aus dem Tractatus von der Logik in die Literatur bzw. von der Philosophie in die Poetologie und verfährt dabei in drei Schritten. Zunächst stellt er alle relevanten Äußerungen Wittgenstein zusammen:

Das Bild ist ein Modell der Wirklichkeit." (Ludwig Wittgenstein, ,Tractatus Logico-Philosophicus', Satz 2.12) Und vorher: „Wir machen uns Bilder von den Tatsachen,“ (Satz 2.1) Weiter aber: „Das Bild besteht darin, daß sich seine Elemente in bestimmter Art und Weise zueinander verhalten.“ (Satz 2.14) „Daß sich die Elemente des Bildes in bestimmter Art und Weise zueinander verhalten [,stellt vor, daß sich die Sachen so zueinander verhalten.] Dieser Zusammenhang der Elemente des Bildes heiße seine Struktur und ihre Möglichkeit die Form der Abbildung." (Satz 2.15 und Satz 2.15111 und 2.1522): Das Bild ist so mit der Wirklichkeit verknüpft; es reicht bis zu ihr. Es ist wie ein Maßstab an die Wirklichkeit angelegt. (150)

Sodann kommentiert er diese Zitatmontage in einem Zwischenschritt, bei dem es vor allem auf den Punkt ankommt, an dem sich das sprachlich erzeugte Welt-Modell der Literatur und die Wirklichkeit berühren. An diesem Punkt

[...] sind wir weit von der Wirklichkeitsignorierung durch die Theoretiker des unmöglichen Romans entfernt. Dagegen sind wir sehr nahe an einer Theorie des Romans als Bild der Wirklichkeit: Er besteht aus Elementen, welche den Elementen der Wirklichkeit (wenn auch nicht unmittelbar) entsprechen; er reicht (über dieZwischenstufe unserer gesamten Erfahrung) bis zur Wirklichkeit; er besteht darin, daß sich seine Elemente zueinander in bestimmter Art und Weise verhalten. Diese Elemente sind wohl die Wörter und Sätze. Aber auch, auf einer zweiten und hintergründigeren Ebene, die Gestalten, die Ereignisse, über die mittels Worten und Sätzen berichtet wird. Das Verhalten dieser Gestalten zueinander, also die Beschaffenheit der Ereignisse, ist das, was innerhalb des Bildes die Struktur der Wirklichkeit entspricht. Es ist auch das, was den Roman ausmacht. (150) 
Man kann in dieser Passage von Dumitrius Essay, nebenbei bemerkt, eine Paraphrase des Kontinuitätsprinzips sehen, das die Idee der Diagrammatik bei Charles Sandes Peirce regiert. ${ }^{13}$ Entscheidend ist die Applikation dieser Idee, die Dumitriu nicht von Peirce, sondern von Wittgenstein übernimmt, auf den Roman also der dritte Argumentationsschritt:

Der Roman besteht darin, daß sich seine Gestalten in bestimmter Art und Weise zueinander verhalten. In diesem Sinne brauchen wir nur weiterzulesen: „Daß sich die Elemente des Bildes in bestimmter Weise zueinander verhalten, stellt vor, daß sich die Sachen so zueinander verhalten. Dieser Zusammenhang der Elemente des Bildes heiße seine Struktur und ihre Möglichkeit seine Form der Abbildung. “ (2.15) Und (2.1511): „Das Bild ist so mit der Wirklichkeit verknüpft; es reicht bis zu ihr." ${ }^{\text {"14 }}$ "Es ist wie ein Maßstab an die Wirklichkeit angelegt.“ (2.1512) Diese Theorie des Wirklichkeitsmodells ist die Theorie der wissenschaftlichen Hypothese, der wissenschaftlichen Erkenntnis, der Erkenntnis überhaupt. Die Theorie jener Wirklichkeitsmodelle also, die wahr oder falsch sind. Es ist auch eine Theorie der Sprache als Mittel zum Bau von wahren oder falschen Wirklichkeitsmodellen. (168)

Nimmt man an dieser Stelle noch einmal den Gedanken auf, dass Wirklichkeitsmodelle heuristische Fiktionen sind, mit deren Hilfe der Mensch die Welt bis auf Weiteres sinnvoll deutet, die aber keineswegs der Weisheit letzter Schluss sind und für

${ }^{13}$ Vgl. Bauer, Matthias / Ernst, Christoph: Diagrammatik Einführung in ein kultur- und medienwissenschaftliches Forschungsfeld. Bielefeld: transcript 2010, S. 25: „Dieses Prinzip besagt, dass die diagrammatische Darstellung respektive Vorstellung in einem unauflöslichen Zusammenhang mit der Realität steht." Es muss gleichsam Schritt für Schritt möglich sein, von ihr zur Wirklichkeit überzugehen.

${ }^{14}$ In diesem letzten, von mir (MB) kursiviertenTeilsatz spricht Wittgenstein als Voraussetzung seiner Logik an, was Peirce mit dem Kontinuitätsprinzip meinte: die Differenz von Zeichen und Objekt, Sprache und Welt ist eingebettet in das Kontinuum eines Denkens, das verbindet und - über Unterscheidungen, Trennungen und Abspaltungen hinweg - Zusammenhänge stiftet. 
sich bestenfalls eine gewisse Wahrscheinlichkeit der Übereinstimmung mit der Wahrheit beanspruchen können, versteht man ohne Mühe, warum Dumitriu im Zuge seiner Aneignung des Tractatusvon Wittgenstein auf Jorge Luis Borges kommt. Denn es geht ihm ja um den Roman und die Erkenntnis-Funktion des Berichts - unabhängig davon, ob der Bericht anhand fiktiver oder realer Ereignis ein Modell der Wirklichkeit entwirft. Dumitriu schreibt:

Es besteht zwischen dem Roman und der Wirklichkeit eben jenes Verhältnis, das nach Wittgensteins Definition das Bild als solches ausmacht, jenes Entsprechen, jene Zuordnung. Borges kommt einer umfassenderen Definition des Romans näher, wenn er sagt, daß ,die Unmöglichkeit, das göttliche Schema zu durchschauen, uns nicht davon abhalten kann, menschliche Schemata zu entwerfen, selbst wenn wir wissen, daß sie bloß vorläufig sind." Es geht also auch darum, jeweils allgemeinste Wirklichkeitsmodelle des menschlichen Lebens zu entwerfen. (172)

Während Borges seine Heuristik im Stil der alten Metaphysik mit Bezug auf Gott formuliert hat, ist es Italo Calvino gelungen, das vermeintliche Absurde der diagrammatischen Operation, vergleichsweise nüchtern, in einem Bonmot festzuhalten: „Die Welt ist nicht lesbar, aber wir müssen gleichwohl versuchen, sie zu entziffern."15 Dumitriu wiederum gelangt zum Schluss seiner Ausführungen zu einer Definition des Romans ,als ludische Berichterstattung über die Gesamtheit der Welt, alsmehrdeutige Wahrheit, im Unterschied zu der von der Wissenschaft abgegebenen, nicht ludischen, sondern theoretischen, eindeutigen Rechenschaft über die Welt, und zwar über einen Teil, eine Ebene, einen Bereich der Welt.“ (249 f.)

Mit dieser Definition bekräftigt er den Anspruch des Romans auf pragmatische Bedeutung (den die Vertreter des

${ }^{15}$ Calvino, Calvino: „Die Welt ist nicht lesbar, aber wir müssen gleichwohl versuchen, sie zu entziffern", in: Zibaldone. Zs. f. ital. Kultur der Gegenwart 1,1 (1986), S. 8-18, hier S. 11. 
,nouveauroman'seinerAnsichtnach preisgeben). Angesprochen wird das alte Paradox, dass die Beschäftigung mit etwas Fiktivem Aufschluss über das Reale geben kann. Die 1965, als Dumitrius Essay erschien, neue Auflösung dieses alten, scheinbaren Widerspruchs lag im Verweis auf die Strukturanalogie von Fiktion und Realität, von Romanfabel und Lebenswelt, imaginiertem und empirischen Handlungsfeld und in der Erkenntnis, dass letztlich alle Ordnungsmuster und Deutungsschema Konstrukte sind, die an der Schnittstelle von Einbildungskraft und Verstand hergestellt werden. „In diesem Sinne“, so hat es Umberto Eco formuliert, ,,arbeiten gewisse Operationen der Kunst, die unserer konkreten Welt so fern zu sein scheinen, letzten Endes darauf hin, uns die imaginativen Kategorien zu liefern, mittels derer wir uns in der Welt bewegen können. "'16

Als Vorwegnahme wesentlicher Merkmale, die später den Begriff der Postmoderne, gerade bei $\mathrm{Eco}^{17}$, ausmachen, und parallel zu den ebenfalls diagrammatischen Überlegungen, die Eco kurz zuvor in Opera aperta (1962) angestellt hatte, entwirft Dumitriu unter Rekurs auf Wittgenstein den Roman somit als ein relationales Modell (Diagramm) der Wirklichkeit, als ein Beziehungsmuster von Handlungen bzw. Handlungsoptionen, als eine Konfiguration, die bedeutsame Schlussfolgerungen auf die Lebenswelt der Leser erlaubt.

\section{Abschließende Bemerkungen}

Aus heutiger Sicht ist die weitgehende Missachtung der Poetik wie der Romane Dumitrius ein drastisches Beispiel für die ,bias' des westeuropäischen Literaturbetriebs, aber auch der Literaturwissenschaft, die sich ausschließlich dem avantgardistischen ,Projekt der Moderne' verschreibt und Südosteuropa

${ }^{16}$ Eco, Das offene Kunstwerk, S. 281.

17 Vgl. Eco, Umberto: Nachschrift zum, Namen der Rose‘. Deutsch von Burkhart Koerber. München: dtv 1986, 76-84. 
ausklammert. Unter den westlichen bzw. den aus Südosteuropa in den Westen emigrierten Intellektuellen galt der ehemalige Kulturfunktionär und Staatspreisträger der Volksrepublik Rumäniens als belastet. Mircea Eliade, Monica Lovinescu und anderewandten sich ausdrücklich gegen eine Publikation von Rendez-vous au jugementdernier im größten Pariser Verlag Gallimard und dagegen, dass Dumitriu die französische Staatsbürgerschaft erhielt.

Für Dumitriu selbst muss es seinerzeit angesichts dessen, was er im Kommunismus erlebt hatte, ein Schock gewesen sein, dass man in der freien Welt meinte, es gebe keine wichtigen Geschichten mehr zu erzählen und der einzige Welt-, Gesellschafts- und Praxisbezug des Romans bestünde in seinem Rekurs auf das Material der Sprache. Seine Auflehnung gegen diese Ideologie des ,nouveauroman' weist den Essay Die Transmoderne als eine nicht nur aus historischen Gründen wichtige Vermittlungsleistung, als einen Kultur-Transfer über Zeiten, Sprachgrenzen und Systemgegensätze hinweg aus, mit dem zu beschäftigen sich nach wie vor lohnt.

$\mathrm{Zu}$ untersuchen bleibt, inwiefern die von Dumitriu nach seiner Flucht aus Rumnänien entwickelte, ,diagrammatische“ Konzeption des Romans eine Umschrift früherer, noch in Rumnänien propagierter Vorstellungen darstellt. Dafür, dass es hier einen genealogischen Zusammenhang geben könnte, spricht zum einen, was Laura Gabriela Laza vom ersten Kongress des Rumänischen Schriftstellerverbands im Jahre 1956 berichtet: Damals thematisierte Dumitriu,

den Unterschied zwischen Naturalismus und sozialistischem Realismus. Der erstere sei die passive Aufnahme der Realitäten, ohne ihre Essenz ansprechen zu wollen. Der letztere hingegen würde anders als der Naturalismus das Typische und die Logik hinter den Sachverhalten suchen. ${ }^{18}$

${ }^{18}$ Laza, „Baumeister war die Angst“, S. 66. 
Zum anderen besteht eine gewisse Ähnlichkeit zwischen der Poetik, die Dumitriu in seinem Essay entwirft, und der Theorie des Romans (1916) von George Lukács, deren entscheidendes Verdienst Lucien Goldmann in seiner vom Marxismus inspirierten Soziologie des modernen Romans (1966) in der Entdeckung homologer Strukturen zwischen Literaturgeschichte und Gesellschaftswandel, Wirtschaftssystem und Kulturprozess sah. ${ }^{19}$ Als Dumitriu, ebenfalls 1966, den 11. Band von Giacomo Casanovas Geschichte meines Lebens mit einem Essay versah, wies er in vergleichbarer Manier aufParallelen zwischen dieser Biographie, dem Tristam Shandy, einem zeitgenössischen Roman von Laurence Sterne, und den Grundzügen des Rokoko hin, das sich von der Geradlinigkeit des Barock befreit habe. „Die Strenge des linearen Barock Versailler Prägung wurde durch eine Legierung mit Spleen, Humor, Perfidie und goldenen Guineen geschmeidig, zuletzt gar formlos gemacht.“20 - „In Giacomos Wesen war nichts Geradliniges. Keine starre Achse“21 - ebenso wenig wie in der von William Hogarths, lineofbeauty“ inspirierten Schlängellinie, die das digressive Prinzip von Sternes Narration nach Art eines Diagramms illustriert. ${ }^{22}$ Die von Dumitriu herausgestellten Strukturanalogien sind nicht der gleichen Geschichtsphilosophie verpflichtet wie die einesLukács oder Goldmann - allein: die Ähnlichkeit der Methode ist evident. Hier wie dort werden Beziehungsmuster aufeinander projiziert, um der Signatur eines Zeitalters auf die Spur zu kommen.

${ }^{19}$ Vgl. Bauer, Romantheorie und Erzählforschung, S. 50 f.

${ }^{20}$ Ciacomo Casanova, Chevalier de Seingalt, Geschichte meines Lebens. Mit einem Essay von Petru Dumitriu ,Casanova oder der Zufällige‘. Herausgegeben und eingeleitet von Erich Loos. Erstmals nach der Urfassung ins Deutsche übersetzt von Heinz von Sauter. Band IX mit zwanzig Farbtafeln Berlin: Propyläen Verlag 1966, S. 7-35, hier S. 9.

${ }^{21}$ Dumitriu, ,Casanova oder der Zufällige‘, S. 11.

${ }^{22}$ Dumitriu, ,Casanova oder der Zufällige', S. 9. 


\section{Literatur}

Aristoteles: Poetik. Übersetzt und kommentiert von Manfred Fuhrmann. Stuttgart: Reclam, 1991.

Bauer, Matthias: Romantheorie und Erzählforschung. Eine Einführung. 2., aktualisierte und erweiterte Auflage. Stuttgart / Weimar, 2005.

Bauer, Matthias / Ernst, Christoph: Diagrammatik Einführung in ein kultur- und medienwissenschaftliches Forschungsfeld. Bielefeld: transcript, 2010.

Calvino, Italo: „Die Welt ist nicht lesbar, aber wir müssen gleichwohl versuchen, siezu entziffern", in: Zibaldone. Zeitschrift für italienische Kultur der Gegenwart 1,1 (1986), S. 8-18.

Camus, Albert: Der Mythos desSisyphos. Ein Versuch über das Absurde. Mit einem kommentierendn Essay von Liselotte Richter. Übertragen von Hans Georg Brenner und Wolfdietrich Rasch. Reinbek beiHamburg: Rowohlt, 1984 [1942].

Camus Albert: Der Mensch in der Revolte. Essays. Aus dem Französischen übertragen von Justus Streller. Neu bearbeitet von Georges Schlucker und Mitarbeit von François Bondy. Reinbek bei Hamburg: Rowohlt 1977, [1951].

Casanova, Giacomo, Chevalier de Seingalt: Geschichte meines Lebens. Mit einem Essay von Petru Dumitriu ,Casanova oder der Zufällige'. Herausgegeben und eingeleitet von Erich Loos. Erstmals nach der Urfassung ins Deutsche übersetzt von Heinz von Sauter. Band IX mit zwanzig Farbtafeln Berlin: Propyläen Verlag, 1966.

Dumitriu, Petru: Treffpunkt Jüngstes Gericht. Aus dem Französischen von Hanns Grössel. Frankfurt am Main: S. Fischer, 1962.

Dumitriu, Petru: Die Transmoderne. Zur Situation des Romans. Frankfurt am Main: S. Fischer, 1965.

Eco, Umberto: Das offene Kunstwerk. Übersetzt von Günter Memmert. Frankfurt am Main: Suhrkamp, 1977 [1962]. 
Eco, Umberto: Nachschrift zum ,Namen der Rose'. Deutsch von Burkhart Kroeber. München: dtv, 1986.

Foucault, Michel: Die Ordnung der Dinge. Eine Archäologie der Humanwissenschaften. Aus dem Französischen von Ulrich Köppen. Frankfurt am Main: Suhrkamp, 1974.

Goldmann, Lucien: Soziologie des modernen Romans. Übersetzt von Lucien Goldmann und Ingeborg Fleischauer. Frankfurt am Main: Suhrkamp, 1984 [1966]

Iser, Wolfgang: „Akte des Fingieren oder Was ist das Fiktive im fiktionalen Text?", in: Funktionen des Fiktiven, hrsg. v. Dieter Henrich und Wolfgang Iser. München: Fink-Verlag, 1983, S. 121-151.

Laza, Laura Gebriela: ,Baumeister war die Angst “. Die politischen Prozesse rumänischer und deutschsprachiger Schriftsteller aus Rumänien nach dem Ungarnaufstand von 1956. Cluj-Napoca: Casa Cărţii de Ştiinţă, 2017.

Lukács, Georg: Die Theorie des Romans. Ein geschichtsphilosophischer Versuch über die Formen der großen Epik. München: dtv, 1994 [1916]

Musil, Robert: „Skizze der Erkenntnis des Dichters“ [1918], in: Robert Musil, Gesammelte Werke in neun Bänden herausgegeben von AdollfFrisé. Band 8. Essays und Reden. Reinbek bei Hamburg: Rowohlt, 1978, S.1025-1030.

Ricour, Paul: Zeit und Erzählung. Band I: Zeit und historische Erzählung. Aus dem Französischen von Rainer Rochlitz. München: Fink-Verlag, 2. Aufl. 2007.

Sartre, Jean-Paul: Was ist Literatur? Übersetzt und herausgegeben von Traugott König. Reinbek bei Hamburg: Rowohlt. 7. Aufl. 1981 [1947].

White, Hayden: Auch Klio dichtet der Die Fiktion des Faktischen. Studien zur Tropologie des historischen Diskurses. Übersetzt von Brigitte Brinkmann-Siepmann. Vorwort von Reinhart Koselleck. Stuttgart: Klett, 1986.

Wittgenstein,Ludwig:TractatusLogico-Philosophicus. LogischPhilosophische Abhandlung. Frankfurt am Main: Suhrkamp, 1962. 